\title{
Causa muy rara de pubertad precoz periférica en una niña: tumor de cordones sexuales con túbulos anulares
}

\author{
A very rare cause of peripheral precocious puberty in a girl: ovarian sex cord \\ tumor with annular tubules
}

\author{
Dra. Julia Sánchez Zahonero ${ }^{a}$, Dr. José A. Ruiz Domingueza , Lic. Sandra Sánchez Zahonero y \\ Prof. Dra. M. José López García
}

\begin{abstract}
RESUMEN
El tumor de los cordones sexuales con túbulos anulares es una neoplasia del estroma gonadal muy infrecuente. Representa el 0,05-0,6\% de todos los tumores ováricos, según series. Se presenta un caso especialmente inusual, en una niña de 6 años, detectado a raíz de una pubertad precoz periférica isosexual. Su interés radica en que no se halló ninguna masa anexa al ovario, sino únicamente una asimetría gonadal, sin signos radiológicos de malignidad.Se realizó una salpingo-ooforectomía unilateral con linfadenectomía pélvica y paraaórtica ipsilateral, por vía laparoscópica, tras confirmarse la presencia de células tumorales en la biopsia intraoperatoria. La evolución posterior de la paciente fue favorable.

Palabras clave: tumores de los cordones sexuales y estroma de las gónadas, pubertad precoz, inhibina B.
\end{abstract}

\begin{abstract}
Sex cord tumor with annular tubules is an extremely uncommon gonadal stromal neoplasm. It represents $0.05-0.6 \%$ of all ovarian tumors, according to series. An unusual case is presented in a 6-year-old girl, detected as a result of an isosexual peripheral precocious puberty. The highlight of this case is that no mass attached to the ovary was found, but only a gonadal asymmetry without radiological signs of malignancy. After confirming the presence of tumoral cells by intraoperative biopsy, unilateral salpingo-oophorectomy with ipsilateral para-aortic and pelvic lymphadenectomy was performed. Afterwards, the evolution of the patient was favorable.
\end{abstract}

Key words: sex cord-gonadal stromal tumors, precocious puberty, inhibin $B$.

http: / / dx.doi.org/10.5546/ aap.2017.e179

Cómo citar: Sánchez Zahonero J, Ruiz Domínguez JA, Sánchez Zahonero $\mathrm{S}$, et al. Causa muy rara de pubertad precoz periférica en una niña: tumor de cordones sexuales con túbulos anulares. Arch Argent Pediatr 2017;115(3):e179-e182.

a. Hospital Clínico Universitario de Valencia (España).

Correspondencia:

Dra. Julia Sánchez Zahonero: ju-san-za@hotmail.com

Financiamiento: Ninguno.

Conflicto de intereses: Ninguno que declarar.

Recibido: 5-10-2016

Aceptado: 9-1-2017

\section{INTRODUCCIÓN}

El tumor de los cordones sexuales con túbulos anulares (TCSTA) es una neoplasia del estroma gonadal extremadamente infrecuente. Constituye el 0,05-0,6\% de todos los tumores ováricos, según series. ${ }^{1-3}$ El primero en describirlo fue Scully ${ }^{4}$ en 1970, y desde entonces, habrá poco más de 150 casos publicados. La serie más amplia es la recogida, en 1982, por Young, la cual incluye 74 casos. $^{5}$

En torno a un 30-40\% de los TCSTA se asocian a síndrome de Peutz-Jeghers (SPJ). Estos casos suelen ser bilaterales, de pequeño tamaño, con calcificaciones y tener un curso más benigno. Por el contrario, cuando no existe tal asociación, son unilaterales, de mayor tamaño, no presentan calcificaciones y pueden mostrar cierto potencial maligno. ${ }^{1,4,5}$

Existen TCSTA descritos en mujeres de entre 4 y 76 años, pero la mayoría surgen en edades reproductivas. ${ }^{1,6,7}$ En raras ocasiones, se presentan en la primera década de la vida, etapa en la que suele diagnosticarse por la aparición de una

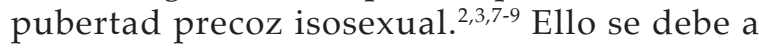
que son tumores productores de hormonas, fundamentalmente, estrógenos y progesterona. ${ }^{10}$

Dada la rareza de estos tumores, en general, y en la edad pediátrica, en particular, comunicar un nuevo caso, en una niña de 6 años, puede ser de utilidad para conocer mejor esta entidad.

\section{CASO CLÍNICO}

Niña de 6 años con telarquia grado 2-3 de Tanner de un mes de evolución, sin antecedentes de interés. Talla diana situada en 0,27 SDE. Exploración física: peso en 0,76 SDE, talla en 1,84 SDE, velocidad de crecimiento en 3,69 SDE, sin vello axilar ni púbico, genitales normales, sin lesiones cutáneas, y el resto de la exploración completamente normal. Edad ósea acorde a la cronológica.

Ante la sospecha de una pubertad precoz, se solicita un análisis hormonal basal con hormona 
luteinizante (luteinizing hormone; LH, por sus siglas en inglés), hormona foliculoestimulante (follicle-stimulating hormone, FSH, por sus siglas en inglés), estradiol, testosterona, androstendiona, dehidroepiandrosterona sulfato, prolactina, hormona estimulante de la tiroides (thyroidstimulating hormone; TSH, por sus siglas en inglés), T4 (tiroxina) libre, un test de LH-RH (factor liberador de gonadotrofinas) (Tabla 1), resonancia magnética (RM) cerebral y una ecografía abdominopélvica. Estos resultados ponen de manifiesto la existencia de una pubertad precoz periférica (imagen cerebral normal, estrógenos elevados con LH y FSH inhibidas). En la ecografía, llama la atención una asimetría ovárica (Figura 1.a), con la impresión diagnóstica de ser un ovario puberal, con folículos normales, sin masas asociadas, hallazgos que se confirman en una segunda exploración ecográfica. A pesar de no observarse una masa clara, se decide realizar una RM abdominopélvica para evaluar con mayor precisión las gónadas y una gammagrafía ósea, por si se tratara de un síndrome de McCune-Albright, en el que están descritas asimetrías ováricas. Asimismo, se repiten estudios analíticos, en los que se incluye la

TABLA 1. Valores de laboratorio

\begin{tabular}{lll}
\hline Hormona & Valor en la paciente & Rango de normalidad del laboratorio \\
\hline LH basal & $<0,1 \mathrm{mUI} / \mathrm{mL}$ & Prepuberal $<1,5 \mathrm{mUI} / \mathrm{mL}$ \\
FSH basal & $<0,1 \mathrm{mUI} / \mathrm{mL}$ & Prepuberal $<0,3 \mathrm{mUI} / \mathrm{mL}$ \\
Estradiol & $22,4 \mathrm{pg} / \mathrm{mL}$ & Prepuberal $<12 \mathrm{pg} / \mathrm{mL}$ \\
Testosterona & $<0,12 \mathrm{ng} / \mathrm{mL}$ & Prepuberal $<0,12 \mathrm{ng} / \mathrm{mL}$ \\
Androstendiona & $<0,3 \mathrm{ng} / \mathrm{mL}$ & $0,02-0,86 \mathrm{ng} / \mathrm{mL}$ \\
Dehidroepiandrostendionasulfato & $22,3 \mathrm{mcg} / \mathrm{dL}$ & $2,5-85,2 \mathrm{mcg} / \mathrm{dL}$ \\
Prolactina & $11,1 \mathrm{ng} / \mathrm{mL}$ & $6-29,9 \mathrm{ng} / \mathrm{mL}$ \\
TSH & $2,75 \mathrm{mcU} / \mathrm{mL}$ & $0,27-4,20 \mathrm{mcU} / \mathrm{mL}$ \\
T4 libre & $1,09 \mathrm{ng} / \mathrm{dL}$ & $0,93-1,79 \mathrm{ng} / \mathrm{dL}$ \\
Test de LH-RH & $\mathrm{LH} \mathrm{basal}<0,1 \mathrm{mUI} / \mathrm{mL}$ & Pico de $\mathrm{LH}>5 \mathrm{mUI} / \mathrm{mL}$ \\
(inmunoquimioluminiscencia) & $\mathrm{LH} \mathrm{a} \mathrm{los} 30 \mathrm{~min}<0,1 \mathrm{mUI} / \mathrm{mL}$ & $($ Resultados sugestivos de pubertad \\
& $\mathrm{LH} \mathrm{a} \mathrm{los} 60 \mathrm{~min} 0,1 \mathrm{mUI} / \mathrm{mL}$ & Precoz central) \\
& $\mathrm{LH} \mathrm{a} \mathrm{los} 120 \mathrm{~min} 0,1 \mathrm{mUI} / \mathrm{mL}$ & $($ Resultados sugestivos de pubertad \\
& $0,6 \mathrm{ng} / \mathrm{mL}$ & precoz periférica) \\
CEA & $<0,61 \mathrm{ng} / \mathrm{mL}$ & $<3,4 \mathrm{ng} / \mathrm{mL}$ \\
$\alpha$-fetoproteína & $16 \mathrm{U} / \mathrm{mL}$ & $0-7 \mathrm{ng} / \mathrm{mL}$ \\
CA-125 & $<0,1 \mathrm{U} / \mathrm{L}$ & $0-35 \mathrm{U} / \mathrm{mL}$ \\
$\beta$-HCG & & $0-1 \mathrm{U} / \mathrm{L}$ \\
\hline
\end{tabular}

LH: hormona luteinizante; FSH: hormona foliculoestimulante; TSH: hormona estimulante de la tiroides; LH-RH: factor liberador de gonadotrofinas humanas; T4: tiroxina; CEA: antígeno carcinoembrionario; $\beta$-HCG: fracción $\beta$ de la gonadotropina coriónica humana; min: minutos.

FIGURA 1. 1.a: Imagen ecográfica del ovario tumoral. 1.b: Imagen del ovario afecto en la resonancia magnética

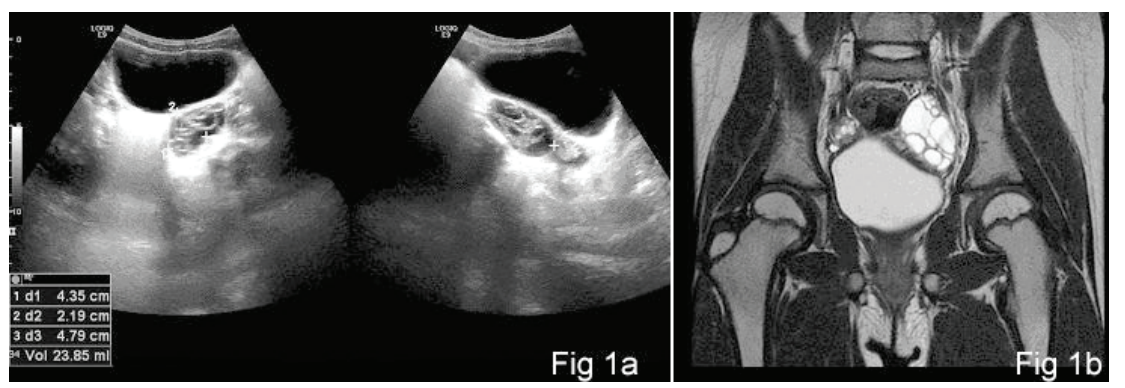

En ambas técnicas de radiodiagnóstico, se aprecia un ovario aumentado de tamaño, pero sin ninguna masa adyacente. 
determinación de marcadores tumorales: antígeno carcinoembrionario (carcinoembryonicantigen; CEA, por sus siglas en inglés), $\alpha$-fetoproteína, CA-125 y gonadotropina coriónica humana $\beta$ ( $\beta$ human chorionic gonadotropin; $\beta$-HCG, por sus siglas en inglés) (Tabla 1 ). En la RM, se informa de la existencia de un ovario izquierdo macroquístico de $39 \times 43 \times 50 \mathrm{~mm}$, con septos finos, sin identificarse masas y ausencia de signos de malignidad (Figura 1.b). Ovario derecho normal. La gammagrafía no revela áreas de displasia ósea y los marcadores tumorales son negativos.

A pesar de no observarse ninguna masa sólida, se decide llevar a cabo una biopsia mediante una laparoscopía. El estudio histológico intraoperatorio informa de un tumor de células de la granulosa, y se efectúauna salpingo-ooforectomía izquierda y linfadenectomía regional.

El análisis anatomopatológico definitivo revela un tumor de cordones sexuales con túbulos anulares. Macroscópico: ovario de $4 \times 2,5 \times 2 \mathrm{~cm}$, sin apreciarse lesión tumoral, con cavidades quísticas de entre 0,3 y $0,8 \mathrm{~cm}$. Microscópico: nidos de formaciones tubulares de características variables. Algunos están compuestos por túbulos bien definidos; otros son más sólidos e indiferenciados. Hay pequeños y múltiples focos de células sueltas que infiltran el estroma ovárico (Figura 2). Inmunohistoquimia: tumor de Wilms (Wilms tumor 1; WT-1, por sus siglas en inglés), vimentina, inhibina, calretinina y receptores para andrógenos positivos. CK-7, 20, 5/6, CA125 , receptores de estrógenos y de progesterona negativos. Trompa y ganglios normales.

Se descarta una asociación con SPJ (estudio genético negativo).

Tras la cirugía, desaparece la telarquia, la velocidad de crecimiento se normaliza y la edad ósea se mantiene acorde con la cronológica.

La paciente se controla cada 3 meses y, por el momento, se encuentra libre de enfermedad.

\section{DISCUSIÓN}

Se comunica un caso de TCSTA no asociado a SPJ que se detectó a raíz de una pubertad precoz periférica isosexual. En los casos pediátricos publicados, ${ }^{2,3,5,8,11}$ las pruebas de imagen mostraban grandes masas sólidas anexas a algún ovario. Sin embargo, en nuestra paciente, no se halló ninguna masa, sino un ovario izquierdo quístico y, aunque grande, no de forma tan llamativa como en lo encontrado en la bibliografía. . $3,5,8,11$ Este hecho ocasionó bastantes dudas a la hora de decidir si someter o no a una intervención a la paciente. Se planteó la posibilidad de que se tratara de un síndrome de McCune-Albright, ya que había casos en los que se describíauna importante asimetría ovárica, ${ }^{12}$ pero, tras quedar descartado por no cumplir los criterios clásicos (sin manchas de café con leche ni displasia ósea), se decidió intervenir. La biopsia intraoperatoria se interpretó como un tumor de células de la granulosa, probablemente, porque los TCSTA eran neoplasias que se encontraban a caballo entre las de las células de la granulosa y las de las células de Sertoli, y podían confundirse en una primera evaluación somera. ${ }^{5}$

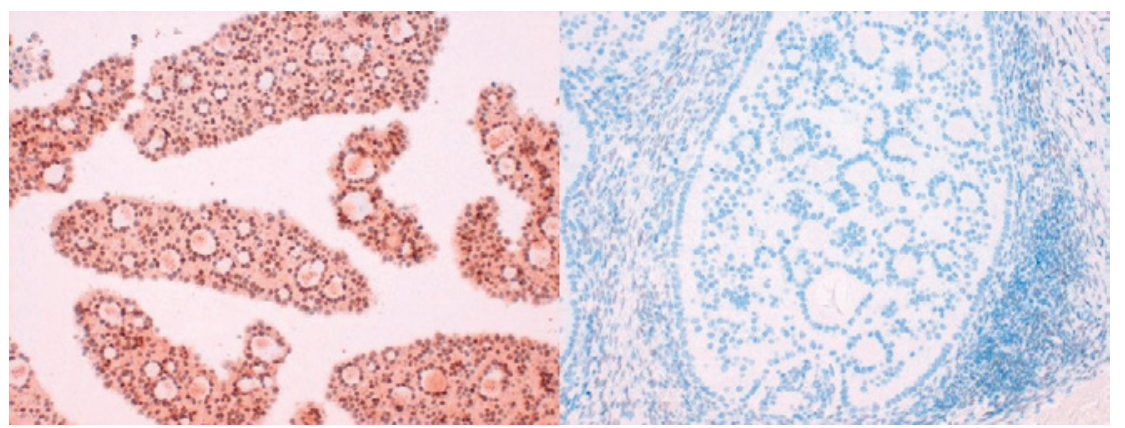

La parteizquierda de la imagen muestra un estudio inmunohistoquímico en el que se aprecian formaciones tubulares de características variables. Algunos nidos están compuestos por túbulos bien definidos, mientras que otros son más sólidos e indiferenciados. Hay pequeños y múltiples focos de células sueltas que infiltran el estroma ovárico. Existe una intensa positividad para receptores de andrógenos. La imagen de la derecha muestra una formación nodular con túbulos ocupados por matriz extracelular y células estromales con débil y aislada positividad a estrógenos. 
Los marcadores tumorales clásicos (CEA, $\alpha$-fetoproteína, CA-125 y $\beta$-hCG) no son de utilidad para diagnosticar este tipo de neoplasias. ${ }^{1-3,5,7,7,11,13,14} \mathrm{Sin}$ embargo, los niveles de inhibina B y la hormona antimülleriana (HAM) se correlacionan bien con la presencia o no de enfermedad. ${ }^{11}$ Esto también las hace válidas para el seguimiento (los valores normales indican ausencia de tumor y su elevación coincide con recidivas). ${ }^{3,13}$ En nuestro caso, puesto que no se sospechó esta rara neoplasia, no se solicitaron tales determinaciones; no obstante, sí se están utilizando en el seguimiento.

En la mayoría de los casos infantiles, el tumor está confinado en el ovario y la salpingoooforectomía unilateral con linfadenectomía pélvica y paraaórtica ipsilateral suele ser curativa. ${ }^{1-3,5,8,11,14}$ No obstante, un $15-20 \%$ de los TCSTA no asociados a SPJ pueden tener un curso desfavorable, con diseminación por vía linfática y con mayor riesgo de recidivas tardías, incluso a los 20 años del diagnóstico. ${ }^{2,3,5,11,14,15}$ Nuestra paciente pertenece a este grupo de TCSTA no asociado a SPJ; por tanto, se tendrá que someter a controles de por vida.

La escasez de casos descritos y su heterogeneidad no han permitido establecer un protocolo concreto de tratamiento para la enfermedad diseminada. ${ }^{5,7}$ Por el mismo motivo, tampoco existen criterios pronósticos claros. Parece haber una correlación entre el tamaño tumoral y el riesgo de evolución maligna. ${ }^{5,14}$ Nuestra paciente no presentaba, al momento del diagnóstico, un tamaño tumoral muy grande $(4,5 \times 2,5 \times 2 \mathrm{~cm})$, en comparación con otros casos pediátricos. ${ }^{2,3,11}$ Por lo que respecta a la histología, existe información contradictoria en cuanto al valor pronóstico del índice mitótico. ${ }^{5,14}$ Un dato llamativo en nuestro caso fue la existencia de múltiples focos de células sueltas que infiltraban el estroma ovárico. Se desconoce el significado pronóstico de este hallazgo, puesto que no se ha encontrado esta descripción histológica en la literatura revisada. En cuanto al estudio inmunohistoquímico, los datos descritos en otros casos son similares a los del nuestro. ${ }^{2}$

Se recomienda un seguimiento estrecho y de por vida de estas pacientes, con determinaciones seriadas de inhibina B y HAM, ${ }^{1-3,5,7,8,11,13,14}$ y pruebas de imagen abdominopélvicas.

\section{CONCLUSIONES}

En niñas con pubertad precoz periférica isosexual que presenten una marcada asimetría ovárica, a pesar de no observarse una masa clara, debe pensarse en este tumor y biopsiar el ovario.

Ante una sospecha de tumor ovárico, además de los marcadores tumorales clásicos, se deben determinar inhibina B y HAM, puesto que sus niveles se correlacionan bien, según lo publicado, con un TCSTA.

\section{Agradecimientos}

Los autores de este artículo quieren mostrar su agradecimiento al Servicio de Anatomía Patológica y de Cirugía Pediátrica del Hospital Clínico Universitario de Valencia.

\section{REFERENCIAS}

1. Sánchez-MéndezJI, Hardisson Hernández DA, Santisteban Padró J, et al. Tumor de los cordones sexuales con túbulos anulares. Aspectos clínicos y morfológicos. Prog Obstet Ginecol 2002;45(5):212-7.

2. Nosov V, ParkS, Rao J, et al. Non-Peutz-Jeghers syndrome associated ovarian sex cord tumor with annular tubules: a case report. Fertil Steril 2009;92(4):1497.e5-8.

3. Ruibal Francisco JL, Palomino Bueno MC, Rivilla Parra F, et al. Sexual precocity in a 7-year old girl, due to a tumor of the ovarian sex cord with annular tubules (SCTAT of Scully). An Esp Pediatr 1998;48(4):409-11.

4. Scully RE. Sex cord tumor with annular tubules a distinctive ovarian tumor of the Peutz-Jeghers syndrome. Cancer 1970;25(5):1107-21.

5. Young RH, Welch WR, Dickersin GR, et al. Ovarian sex cord tumor with annular tubules: review of 74 cases including 27 with Peutz-Jeghers syndrome and four with adenoma malignum of the cervix. Cancer 1982;50(7):1384-402.

6. Scully RE. Hormonally active ovarian tumors. Verh Dtsch Ges Pathol 1997;81:245-52.

7. Qian Q, You Y, Yang J, et al. Management and prognosis of patients with ovarian sex cord tumor with annular tubules: a retrospective study. BMC Cancer 2015;15:270.

8. Shen K, Wu PC, Lang JH, et al. Ovarian sex cord tumor with annular tubules: a report of six cases. Gynecol Oncol 1993;48(2):180-4.

9. Zumkeller W, Krause U, Holzhausen HJ, et al. Ovarian sex cord tumor with annular tubules associated with precocious puberty. Med Pediatr Oncol 2000;35(2):144-6.

10. Dolan J, Al-Timimi AH, Richards SM, et al. Does ovarian sex cord tumour with annular tubules produce progesterone? J Clin Pathol 1986;39(1):29-35.

11. Mantrana E, Carrasco L, Garrido R. Tumor de los cordones sexuales con túbulos anulares. Prog Obstet Ginecol 2000;43(8):423-5.

12. Foster CM, Feuillan P, Padmanabhan V, et al. Ovarian function in girls with McCune-Albright syndrome. Pediatr Res 1986;20(9):859-63.

13. Gustafson ML, Lee MM, Scully RE, et al. Müllerianinhibiting substance as a marker for ovarian sex-cord tumor. $N$ Engl J Med 1992;326(7):466-71.

14. Meyerson D, Cuello M, Brañes J, et al. Tumor delos cordones sexuales con túbulos anulares del ovario no asociado a síndrome de Peutz-Jeghers: reporte de un caso. Rev Chil Obstet Ginecol 2006;71(1):57-62.

15. Roth LM. Recent advances in the pathology and classification of ovarian sex cord-stromal tumors. Int J Gyneco lPathol 2006;25(3):199-215. 